Published in final edited form as:

$H G G$ Adv. 2021 April 8; 2(2): . doi:10.1016/j.xhgg.2021.100026.

\title{
Genotype and defects in microtubule-based motility correlate with clinical severity in KIF1A-associated neurological disorder
}

\author{
Lia Boyle ${ }^{1}$, Lu Rao ${ }^{2}$, Simranpreet Kaur ${ }^{3}$, Xiao Fan ${ }^{1,4}$, Caroline Mebane ${ }^{1}$, Laura Hamm ${ }^{5}$, \\ Andrew Thornton ${ }^{6}$, Jared T. Ahrendsen ${ }^{7}$, Matthew P. Anderson ${ }^{7,8,9,10}$, John \\ Christodoulou $^{3}$, Arne Gennerich ${ }^{2}$, Yufeng Shen ${ }^{4,11}$, Wendy K. Chung ${ }^{1,12,{ }^{*}}$ \\ ${ }^{1}$ Department of Pediatrics, Columbia University Irving Medical Center, New York, NY 10032, USA \\ ${ }^{2}$ Department of Anatomy and Structural Biology and Gruss Lipper Biophotonics Center, Albert \\ Einstein College of Medicine, Bronx, NY 10461, USA \\ ${ }^{3}$ Murdoch Children's Research Institute, Parkville, Department of Pediatrics, University of \\ Melbourne, Melbourne, VIC 3052, Australia \\ ${ }^{4}$ Department of Systems Biology, Columbia University Irving Medical Center, New York, NY \\ 10032, USA
}

${ }^{5}$ Genetic \& Genomic Sciences, Icahn School of Medicine at Mount Sinai, New York, NY 10029 , USA

${ }^{6}$ Columbia University Vagelos College of Physicians and Surgeons, New York, NY 10032, USA

${ }^{7}$ Department of Pathology, Beth Israel Deaconess Medical Center, Boston, MA 02215, USA

${ }^{8}$ Department of Neurology, Beth Israel Deaconess Medical Center, Boston, MA 02215, USA

${ }^{9}$ Boston Children's Hospital Intellectual and Developmental Disabilities Research Center, 300 Longwood Avenue, Boston, MA 02115, USA

${ }^{10}$ Program in Neuroscience, Harvard Medical School, 300 Longwood Avenue, Boston, MA 02115, USA

${ }^{11}$ Department of Biomedical Informatics, Columbia University Irving Medical Center, New York, NY 10032, USA

${ }^{12}$ Department of Medicine, Columbia University Irving Medical Center, New York, NY 10032, USA

\footnotetext{
This is an open access article under the CC BY-NC-ND license (http://creativecommons.org/licenses/by-nc-nd/4.0/).

*Correspondence: wkc15@cumc.columbia.edu.

Supplemental information

Supplemental information can be found online at https://doi.org/10.1016/j.xhgg.2021.100026.

Declaration of interests

The authors declare no competing interests.

Web resources

Agilent, https://www.agilent.com/genomics/qcpd

Autism Brain Net, https://www.autismbrainnet.org/

Exome Sequencing Project, https://evs.gs.washington.edu/EVS/

OMIM, https://www.omim.org/

SnapGene Viewer software, https://www.snapgene.com/snapgene-viewer/
} 


\section{Summary}

KIF1A-associated neurological disorder (KAND) encompasses a group of rare neurodegenerative conditions caused by variants in $K I F 1 A$, a gene that encodes an anterograde neuronal microtubule (MT) motor protein. Here we characterize the natural history of KAND in 117 individuals using a combination of caregiver or self-reported medical history, a standardized measure of adaptive behavior, clinical records, and neuropathology. We developed a heuristic severity score using a weighted sum of common symptoms to assess disease severity. Focusing on 100 individuals, we compared the average clinical severity score for each variant with in silico predictions of deleteriousness and location in the protein. We found increased severity is strongly associated with variants occurring in protein regions involved with ATP and MT binding: the P loop, switch I, and switch II. For a subset of variants, we generated recombinant proteins, which we used to assess transport in vivo by assessing neurite tip accumulation and to assess MT binding, motor velocity, and processivity using total internal reflection fluorescence microscopy. We find all modeled variants result in defects in protein transport, and we describe three classes of protein dysfunction: reduced MT binding, reduced velocity and processivity, and increased non-motile rigor MT binding. The rigor phenotype is consistently associated with the most severe clinical phenotype, while reduced MT binding is associated with milder clinical phenotypes. Our findings suggest the clinical phenotypic heterogeneity in KAND likely reflects and parallels diverse molecular phenotypes. We propose a different way to describe KAND subtypes to better capture the breadth of disease severity.

\section{Introduction}

$K I F 1 A$-associated neurological disorder (KAND) (KIF1A [MIM: 601255]) encompasses a recently identified group of rare progressive neurodegenerative conditions caused by pathogenic variants in KIF1A. ${ }^{1-42}$ Variants can be inherited dominantly or recessively, with de novo variants associated with the most severe phenotypes. An important feature of KAND is the large number of disease-causing variants, predominantly missense variants within the motor domain of the protein. In the existing literature, 71 variants have been described to date, and here we describe 42 additional variants. ${ }^{2-34,36-39,41-43}$ More than $30 \%$ of individuals with KAND have private variants, and there are likely many more variants that remain to be identified. KAND has a broad phenotypic spectrum, which can include spasticity, neurodevelopmental delay, intellectual disability, autism, microcephaly, progressive spastic paraplegia, autonomic and peripheral neuropathy, optic nerve atrophy, cerebral and cerebellar atrophy, and seizures. ${ }^{2-34,36-39,41-43}$

At the cellular level, KIF1A is a neuron-specific member of the kinesin-3 family of ATPdependent microtubule (MT) molecular motor proteins. It predominately functions as a dimer and is responsible for anterograde transport of multiple cargos, including synaptic vesicle precursors and dense core secretory vesicles on axonal and dendritic MTs. ${ }^{44,45}$ The progression of kinesins along MTs requires ATP binding and hydrolysis, which cause conformational changes within the motor domain that power the forward motion of the motor. ${ }^{44}$ 
KIF1A has been associated with two different autosomal recessive conditions: hereditary spastic paraplegia (HSP, MIM: 610357) and hereditary sensory and autonomic neuropathy (HSAN, MIM: 614213). ${ }^{2,3}$ Individuals with biallelic missense variants in the motor domain were found to have HSP $30,{ }^{3}$ a condition consisting of peripheral nerve degeneration and severe distal sensory loss with distal motor involvement.

Heterozygous variants in KIF1A have been associated with NESCAV syndrome (neurodegeneration and spasticity with or without cerebellar atrophy or cortical visual impairment, formerly mental retardation autosomal dominant 9) (MIM: 614255), PEHO syndrome (progressive encephalopathy with edema, hypsarrhythmia, and optic atrophy), and inherited cases of both simple and complicated HSP. ${ }^{4-34,36-39,41-43}$ More recently, individuals with clinically diagnosed Rett syndrome were identified with de novo KIF1A variants in individuals who did not have variants in the genes that predominantly cause Rett syndrome (MECP2, CDKL5, and FOXG1). ${ }^{35,40}$

Here we describe the natural history of KAND in 117 individuals with 68 different variants in KIF1A, provide detailed medical and neurodevelopmental phenotypes, and demonstrate a wide range of clinical severity with associated neuropathology on autopsy. We generated a KAND clinical severity score and correlate the clinical phenotype with genotype and molecular phenotype of the KIF1A motor.

\section{Subjects and methods}

\section{Cohort}

The procedures followed were in accordance with the ethical standards of the responsible committee on human experimentation. This study was approved by the Institutional Review Board of Columbia University, and proper informed consent was obtained for all participants. Individuals were referred by the family organization KIF1A.org or their physicians. Clinical genetic test reports were reviewed to verify the diagnosis. Affected individuals or their parents or legal guardians completed a detailed structured medical history interview by phone with some medical record verification. The Vineland Adaptive Behavior Scales, Third Edition (VABS-3) was administered by computer. In a smaller subset of individuals, the Vineland Adaptive Behavior Scales, Second Edition (VABS-II) was also completed as a structured telephone interview. The VABS-3 is a standardized assessment of adaptive behavior designed to be used for individuals of all ages with developmental, intellectual, or other disabilities to assess adaptive behavior in four domains: communication, daily living skills, socialization, and motor skills. Domain-level scores for three of the domains (communication, daily living skills, and socialization) are used to generate an overall Adaptive Behavior Composite score, a number between 20 and 140. To assist with interpretation of scores, VABS-3 scores of $>85$ correspond with adequate or above levels of adaptive behavior, with scores in the 71-85 range corresponding with moderately low levels and scores $<71$ corresponding with low levels. The VABS-II Adaptive Behavior Composite score is calculated similarly but also incorporates the motor skills domain. VABS-II Adaptive Behavior Composite scores can be interpreted as corresponding to adaptive behavior levels of average or above average ( $\$ 85$ ), borderline (70-84), mild (55$69)$, moderate (40-54), severe (25-39), and profound (20-24). Clinical records and imaging 
data were reviewed to verify participant/caregiver reported history. Study data were managed using REDCap electronic data capture tools hosted at Columbia University Mailman School of Public Health. ${ }^{11,12}$

In two individuals, postmortem brain specimens were collected through the Autism Brain Net. Consent for autopsy was obtained from the decedent's next of kin, which included provisions to save tissue for diagnostic and research purposes. The brain and spinal cord were removed and placed directly into $10 \%$ neutral buffered formalin fixed for 14 days before gross examination and sectioning. Formalin-fixed paraffin-embedded tissue was sectioned and stained with $\mathrm{H} \& \mathrm{E}$ and Luxol fast blue (H\&E/LFB) prior to histologic examination by two neuropathologists.

\section{Genetic data analysis}

Individuals received clinical genetic testing from multiple clinical laboratories, with some individuals receiving whole-exome sequencing and others panel gene testing. All KIF1A variants were re-interpreted centrally using ACMG (American College of Medical Genetics and Genomics) guidelines. ${ }^{46}$ Genetic reports were also reviewed for any additional potentially contributing genetic factors that might contribute to the phenotype (Tables S1 and S2).

A standardized transcript (GenBank: NM_001244008.2) and Human Genome Variation Society (HGVS) were used for nomenclature of all variants. Variants previously described in the literature were all standardized to this transcript. Variants were annotated with their population allele frequencies in Exome Aggregation Consortium (ExAC), ${ }^{47}$ Exome Sequencing Project (ESP), 1000 Genomes Samples, ${ }^{48}$ and Genome Aggregation Database (gnomAD). ${ }^{47}$ Computational scores from REVEL (Rare Exome Variant Ensemble Learner) and CADD (Combined Annotation Dependent Depletion) version 1.4 were collected using ANNOVAR version 2018Apr16. Biallelic disease-associated variants that fit to an autosomal recessive inheritance pattern were identified. One individual was mosaic for a missense variant, and she was therefore excluded from the analyses to assess severity score, though her variant and her disease severity are shown in Figure 1.

\section{Computational methods}

To summarize clinical severity, we used numerical values to represent the clinical features of brain atrophy, optic atrophy, seizure, microcephaly, developmental delay, spasticity, and hypotonia (yes [1], no [-1], and not sure [0]), and converted VABS composite score to 1 for VABS scores $<86$ and -1 for scores $>85$. VABS-3 Adaptive Behavior Composite score was used in all except three individuals where VABS-3 data were not available; in those individuals VABS-II data were available, and so in those instances the VABS-II data Adaptive Behavior Composite Scores were used instead. While direct comparison of VABSII and VABS-3 data is not generally recommended, since a given individual typically scores lower on VABS-3 at the same time point, in this case all three individuals had VABS-II scores well below the severity score cutoff of 85 . We designed a heuristic severity score by weighted sum of the medical history data. We set the weight to 2 for brain atrophy, seizure, and microcephaly, and the rest were given a weight of 1 . We further performed a principal 
component analysis (PCA) in individuals who had both medical history and VABS data. We characterized the genetic variants using their corresponding locations in different functional region of the protein, particularly ATP and MT binding regions.

\section{Construct generation}

A recombinant $H$. sapiens KIF1A(1-393)-LZ-SNAPf-EGFP-6His construct containing the motor domain (amino acids 1-361), neck linker, neck coil domains, and a GCN4 leucine zipper (LZ) for stabilized dimerization was used for total internal reflection fluorescence (TIRF) microscopy. To generate this construct, a $R$. norvegicus KIF1A(1-393)-LZ-mScarlet construct obtained from Dr. Kassandra M. Ori-McKenney ${ }^{49}$ (UC Davis) was modified as follows: KIF1A(1-393) was amplified by PCR and inserted into a vector with a SNAPf-tag, GFP-tag, and a 6xHis tag at the C terminus.H. sapiens $\mathrm{KIF} 1 \mathrm{~A}(1-393)$ is identical to $R$. norvegicus, except for Ile 359, which is Val in $H$. sapiens. The NEB Q5 site-directed mutagenesis kit (New England Biolabs, \#E0554S) was therefore used to induce the c.1075A $>$ G (p.I359V) mutation to generate the H. sapiens KIF1A(1-393)-LZ-SNAPfEGFP-6His construct. This KIF1A construct was then used as template to generate the 8 aberrant motors using the Q5 mutagenesis kit. All constructs were confirmed by sequencing.

For neurite tip accumulation assays, a KIF1A(1-393)-LZ-3xmCit construct tagged with three tandem mCitrine (mCit) fluorescent proteins at the $\mathrm{C}$ terminus was obtained from Dr. Kristen Verhey (University of Michigan, MI, USA).$^{50}$ The sequences of all the constructs used in this project were analyzed using SnapGene Viewer software (version 4.0.2; GSL Biotech). Primers flanking individual specific variation site were designed using web-based QuikChange Primer Design Program available online as per the software instructions. Variants observed in affected individuals were then introduced into the KIF1A(1393)-3xmCit construct using QuikChange lightning mutagenesis kit (Agilent Technologies, \#210519) according to the manufacturer's instructions.

Cell lysate-based single-molecule TIRF assay-Expression of KIF1A constructs in E. coli bacteria for single-molecule TIRF imaging is described in detail in the Supplemental material. Following the generation of the $E$. coli cell lysate and its clearance by centrifugation, the cleared lysate was used for the initial single-molecule TIRF microscopy studies to determine whether the KIF1A motors generated from mutant transcripts are mobile or not. The assay was performed as described before, ${ }^{51}$ except that a different assay buffer was used (see Supplemental material). The lysate was diluted 100-fold in the assay buffer before the solution was flown into theslide chamber. The GFP tag was used to track the motors. 600 frames were acquired for each video, with an acquisition time of $200 \mathrm{~ms}$ per frame. Kymographs were generated using Fiji.

Protein purification-Further protein purification was performed as described in detail in the Supplemental material. Following the incubation of the cleared $E$. coli cell lysate with an Ni-NTA resin, the resin was washed and then labeled with a SNAP-Cell TMR-Star ligand. After another wash step, the protein was eluted with elution buffer and then flash frozen and stored at $-80^{\circ} \mathrm{C}$. Before further usage, an MT binding and release (MTBR) assay was 
performed as previously described, ${ }^{52}$ with slight modifications (see Supplemental material). The resulting protein solution was aliquoted and flash frozen as the "MTBR" fraction.

Single-molecule TIRF assay with purified proteins-For the wild-type (WT) and mobile motors, MTBR fractions were used for the single-molecule TIRF assay, and the dilutions were adjusted to an appropriate density of motors on MTs. The TIRF assay was performed as described above for the studies with the lysate, except TMR was used to track the motors. Images were analyzed using home-build MATLAB software, and graphs were generated using Prism.

Neurite tip accumulation-SH-SY5Y (human neuroblastoma) cell lines were grown in RPMI growth media (Sigma; Cat. no. R0883; with $3.7 \mathrm{~g} / \mathrm{L} \mathrm{NaHCO}_{3}, 2.0 \mathrm{~g} / \mathrm{L}$ glucose, and $0.3 \mathrm{~g} / \mathrm{L} \mathrm{L}$-glutamine) supplemented with $10 \%$ (vol/vol) fetal bovine serum (FBS), $1 \times$ PenStrep at $37^{\circ} \mathrm{C}$ in a humidified incubator and $5 \% \mathrm{CO}_{2}$. The optimized density of SH-SY5Y cells $\left(5 \times 10^{4}\right.$ cells per well) was plated on sterile glass coverslips coated with $3.3 \%$ rat collagen (Corning; Cat. no.354236) and 2\% Matrigel (Corning; Cat. no. 354248) in RPMI media without serum and antibiotics in a 12-well plate. After $24 \mathrm{~h}$, cells were transfected with the appropriate construct using Lipofectamine 2000 (Life Technologies; Cat. no. $11668019)$ according to the manufacturer's instructions. Neuronal differentiation of the transfected cells was then induced using RPMI differentiation media (RPMI supplemented with $1 \%$ FBS, $1 \times$ Pen-Strep, and $10 \mu \mathrm{M}$ retinoic acid [RA; Sigma; R2625]) that was refreshed every $24 \mathrm{~h}$ for 3 days. Cells were then washed with warm $1 \times$ phosphate-buffered saline (PBS; $137 \mathrm{mM} \mathrm{NaCl}, 5.4 \mathrm{mM} \mathrm{KCl}, 1.28 \mathrm{mM} \mathrm{NaH}_{2} \mathrm{PO}_{4}, 7 \mu \mathrm{mM} \mathrm{Na}_{2} \mathrm{HPO}_{4}[\mathrm{pH}$ 7.4]),fixed in 4\% (vol/vol) paraformaldehyde (PFA; Sigma; Cat. no. 158127) in PBS for 20 $\mathrm{min}$ at room temperature before mounting on glass slides using Prolong Gold with DAPI (Thermo Fisher Scientific; Cat. no. P36935). Images were acquired using LSM780 fluorescence microscope with $40 \times$ objective (zoom factor $=2$ ). ImageJ was used to quantify the mean florescence intensity (MFI) of the expressed fluorescent protein along the neurite length and cell body of cells. Statistical analysis on the average MFI was performed using Mann Whitney in Prism software (GraphPad). All experiments were performed in triplicate and repeated at least two times prior to data analysis.

Structure rendering-All structures were rendered using Chimera. ${ }^{46}$ The structure depicted is PDB: 4UXP, H. sapiens KIF1A cryo-EM structure in the presence of AMP-PNP. ${ }^{53}$ PDB: $2 \mathrm{HXF}^{54}$ (cryo-EM structure of the M. musculus MT-bound KIF1A motor domain) was used to assign the secondary structure to the residues.

\section{Results}

\section{Summary of clinical phenotypes}

A summary of the clinical, radiological, and neurodevelopmental features of individuals is shown in Table 1, with specific variant and severity information available in Table S1. We report 117 individuals, of whom we have complete phenotypic data on 100 (Figures 1 and S1; Table S1). While the majority of individuals in our cohort had heterozygous KIF1A variants (115/117), we describe two biallelic individuals with asymptomatic carrier parents. 
In each biallelic instance there was one variant within the motor domain and one outside the motor domain. Among our heterozygous individuals, the majority of $K I F 1 A$ variants (64/115) were de novo, and 3 individuals were inherited from an affected parent. Clinical information is not available for those affected parents. In some individuals (48/115), parental testing results were not available. In a single instance, the affected individual was mosaic for the variant in blood.

Our cohort includes 47 females and 53 males, with age at interview spanning 6 months to 39 years old, with a mean age of 9.9 years and a median of 7.3 years. The reported ages of onset range from birth to 9 years old (mean: 11 months; median: 6 months). The most frequently observed symptoms in our cohort were hypotonia $(84 \%, 84 / 100)$ and hypertonia or spasticity $(81 \%, 81 / 100)$. Nearly all individuals had some degree of developmental delay and/or intellectual disability $(92 \%, 92 / 100)$. Among those who had neuroimaging, the majority $(58 \%, 54 / 93)$ had abnormal findings, most commonly cerebellar atrophy $(35 \%$, $33 / 93)$, abnormalities in the corpus callosum $(11 \%, 10 / 93)$, and cerebral atrophy $(6 \%, 6 / 93)$. Half of our cohort (50/100) had optic nerve atrophy or optic nerve hypoplasia, and there was a high prevalence of cortical visual impairment $(20 \%, 20 / 100)$ and strabismus $(26 \%$, 26/100).

Developmentally, most individuals were globally delayed, with an average VABS-3 Adaptive Behavior Composite score of 60.6, which corresponds with low levels of adaptive functioning (population mean of 100, SD of 15) (Tables 1 and S3). Frequently reported neurobehavioral diagnoses included autism $(20 \%, 16 / 80)$, attention deficit hyperactivity disorder $(24 \%, 19 / 80)$, anxiety $(19 \%, 15 / 80)$, and obsessive-compulsive disorder $(5 \%, 4 / 80)$. We also saw some features commonly seen in Rett syndrome, including stereotypic hand movements like wringing or clasping $(21 \%, 17 / 80)$, small extremities $(34 \% 27 / 90)$, cold extremities $(60 \%, 48 / 80)$, bruxism $(35 \%, 28 / 80)$, abnormal laughing spells $(15 \%, 11 / 80)$, scoliosis $(14 \%, 14 / 100)$, and high pain tolerance $(65 \%, 52 / 80)$.

Seizures were common in our cohort, with $42 \%$ (42/100) reporting a history of seizures. Multiple seizure types were described, often in the same individual, with absence seizures as the most common in 29\% (29/100) of the cohort overall and in 69\% (29/42) of those with seizures.

We saw a number of symptoms that may be features of dysautonomia. Just under half of individuals $(46 \%, 37 / 80)$ experience difficulty with temperature regulation, including sporadic fevers unrelated to illness. Gastrointestinal issues were common, with $40 \%$ (40/100) reporting gastroesophageal reflux disease (GERD), constipation (39\%, 39/100), and diarrhea $(17 \%, 17 / 100)$. Urinary retention and urinary incontinence were both reported. Many affected individuals experienced excessive salivation $(26 \%, 21 / 80)$ or problems swallowing $(29 \%, 23 / 80)$. Ten percent of the overall cohort (10/100) required enteric nutritional support, in many individuals due to the difficulty swallowing. While these symptoms may reflect autonomic dysfunction, there may be other underlying causative factors, and further direct assessment of autonomic function will be beneficial. 
We saw additional features not previously described, including a small number of individuals with abnormalities in the urogenital system. Irregularities in external genitalia were seen in both males and females (10/53 males, 4/47 females), and a few individuals had structural renal issues, including renal agenesis, hydronephrosis, medullary nephrocalcinosis, and urinary reflux. Another feature not previously reported in KAND was short stature, which we observed in 11\% (11/100) of our cohort. In three of those individuals, complete growth hormone deficiency was noted, which has been previously observed. ${ }^{6,31,35}$

The condition is neurodegenerative, though the nature and course of decline varied by individual. Spasticity was progressive, and a number of individuals required surgical intervention: 15\% (15/100) received tendon lengthening procedures. By the time they reached their 20s, many individuals were largely wheelchair dependent. When present, optic nerve atrophy also appeared to be progressive in most individuals. While the loss of vision and of motor skills limited an individual's ability to participate in the activities of daily living over time, we largely did not see cognitive regression.

\section{Neuropathological findings}

At autopsy of a 13-year-old female with a c.296C >T (p.Thr99Met) variant, the cerebellum was severely atrophic, most prominently in the midline, with relatively normal gyral patterning and development of the cerebrum (Figures 2A-2F). Microscopic examination revealed severe cerebellar atrophy most prominent in the superior cerebellar vermis, including a thin molecular layer, a depletion of Purkinje and internal granule cells, Bergmann gliosis, and white matter pallor. Rare axonal spheroids were observed. The dentate nucleus as well as the inferior olivary nucleus of the medulla showed marked loss of neurons and severe reactive gliosis. Residual neurons in these areas were hypereosinophilic with pyknotic nuclei. Autopsy was also performed for a second individual with a de novo c.296C>T (p.Thr99Met) variant, a 5-year 9-month-old female, and features were similar across both individuals (Figures 2G-2I).

\section{Computational results}

Our heuristic severity score approximated a normal distribution across all individuals (Figure 3A). Using all available medical history and VABS data, we limited the analyses to age, sex, and common symptoms ( $>15 \%$ prevalence in the cohort), with $<10 \%$ missing data. PCA showed principal component 1 (PC1) was attributed mostly to the same symptoms used in the heuristic severity score as well as the VABS scores. PC2 is correlated with the age of disease onset. Individuals with earlier-onset disease were more likely to develop microcephaly, and individuals with later-onset disease more often underwent musculoskeletal surgeries. The first three PCs explained 13.5\%, 7.8\%, and 5.8\%, respectively, of the variance in the data. Additionally, we found that the heuristic severity score was highly correlated $(\mathrm{R}=0.824)$ with PC1 (Figure 3B). Since PCA is an unsupervised analysis, this high correlation supported the conclusion that the heuristic score is a good overall measurement of KAND severity. 
We hypothesized that severely affected individuals have genetic variants with similar characteristics. We did not observe significant correlation between severity score and prediction score for variant deleteriousness, such as REVEL and CADD, but all CADD scores were larger than 20 in this dataset. More importantly, the severity score was strongly associated with location in regions of the protein involved with ATP and MT binding: the P loop, switch I, and switch II (Figure 3D). We found that 28 out of 33 (84.8\%) of the variants seen in individuals with the most severe KAND (score 25 ) corresponded to residues located in the ATP and MT binding regions, whereas only 45 out 69 (65.2\%) of the remaining individuals had variants in these same regions (odds ratio $=1.22$, $\mathrm{p}$ value $=0.04$ ).

Additionally, individuals with variants corresponding to the ATP and MT binding regions have significantly higher severity scores $(\mathrm{p}=0.05$; delta mean $=1.8)$ than the ones with variants outside the ATP and MT binding regions (Figure 3C).

In a progressive condition, severity would be expected to increase over the individual's lifetime, but we currently have cross-sectional and retrospective recall rather than prospective data. We observe a significant relationship between age at interview and severity score. Simple linear regression suggests age explains less than $10 \%$ of the variation in severity score $\left(\mathrm{t}=2.61, \mathrm{p}=0.00962, \mathrm{R}^{2}=0.066\right)$. The age distribution across the three severity groupings (mild: $>1$; moderate: $1-4$; severe: $<4$ ) shows individuals with mild scores are relatively younger, while individuals in the moderate and severe ranges have a similar age distribution (Figure S3).

Effect of KAND variants on neurite tip accumulation-To assess the effects of KAND variants on overall motor function of KIF1A, we expressed WT and mutant KIF1A (1-393) tagged with three tandem copies of fluorescent monomeric citrine (mCit) in retinoic acid-differentiated neuronal SH-SY5Y cells and measured the MFI at the cell body and along the neurite length. Consistent with previous work from our group and others, WT KIF1A accumulated at the distal tip of growing neurites in differentiated SH-SY5Y cells $^{35,50}$ (Figure 4). All aberrant KIF1A motors tested showed significant abilities to accumulate along the neurites of differentiated cells.

Effect of KAND variants on velocity and processivity-To determine the effects of KAND variants on the velocity and processivity (the ability to take multiple steps before dissociating) of KIF1A, we employed TIRF microscopy and a tail-truncated, constitutively active KIF1A (H. sapiens amino acids [aa] 1-393) with a C-terminal leucine zipper (LZ) for stabilized dimerization as well as a SNAP-tag and a GFP tag. Motor constructs generated from WT and mutant KIF1A were expressed in E. coli bacteria. Our recent work has shown that the velocity and force-generation properties of KIF1A expressed in E. coli bacteria and mammalian cells are statistically indistinguishable. ${ }^{55} \mathrm{~A}$ similar construct has also been used in other studies. ${ }^{49,56,57}$

Eight variants were selected to represent a range of associated clinical disease severity, and protein constructs modeling the most commonly occurring variants were generated by mutagenesis. The positions of the mutations are illustrated in Figure 5. The residues p.Arg13His (c.38G >A), p.Thr99Met (c.296C >T), p.Gly251Arg (c.751G>A), p.Glu253Lys (c.757G >A), and p.Arg254Trp (c.760C $>$ T) are at or close to the ATP binding site, while 
p.Ser274Leu (c.821C >T), p.Pro305Leu (c.914C > T), and p.Arg316Trp (c.946C >T) are near the protein's MT binding site, specifically at the $\beta$-tubulin interaction interface (Figure $5 \mathrm{~A}$ ). For an initial assessment of the effects of the mutations, we used the $E$. coli cell lysate with the expressed KIF1A motors without further purification and directly performed singlemolecule TIRF microscopy studies. These initial experiments revealed that while the p.Arg254Trp (c.760 C>T), p.Ser274Leu (c.821 C>T), p.Pro305Leu (c.914C>T), and p.Arg316Trp (c.946C >T) motors retained their ability to move processively, they appeared to have reduced run lengths (average distance traveled) (Figure 5B). In contrast, the p.Arg13His (c.38G>A), p.Thr99Met (c.296C>T), p.Gly251Arg (c.751G>A), and p.Glu253Lys (c.757G $>A$ ) motors were completely unable to move (Figure 5B). We found p.Thr99Met (c.296C>T), p.Gly251Arg (c.751G>A), and p.Glu253Lys (c.757G >A) attach to MTs in rigor, while p.Arg13His (c.38G $>$ A) lost its ability to bind to MTs (Figure 5B).

To further quantify the motility behaviors of the motile aberrant motors, p.Arg254Trp (c.760C $>$ T), p.Ser274Leu (c.821C > T), p.Pro305Leu (c.914C >T), and p.Arg316Trp (c.946C $>$ T) were purified via Ni-NTA affinity purification and labeled with a SNAP-tag TMR ligand, followed by MTBR affinity purification. ${ }^{58}$ All four aberrant motors exhibited reduced velocities. While WT KIF1A has an average velocity of $2.10 \pm 0.03 \mu \mathrm{m} / \mathrm{s}$ (mean \pm SEM), p.Arg254Trp (c.760C >T), p.Ser274Leu (c.821C >T), p.Pro305Leu (c.914C >T), and p.Arg316Trp (c.946C >T) move at $1.0 \pm 0.01 \mu \mathrm{m} / \mathrm{s}, 0.7 \pm 0.08 \mu \mathrm{m} / \mathrm{s}, 1.1 \pm 0.01 \mu \mathrm{m} / \mathrm{s}$, and 0.8 $\pm 0.01 \mu \mathrm{m} / \mathrm{s}$, respectively (Figure 5C). In addition, all four aberrant motors exhibited reduced processivities. While WT KIF1A is processive with a run length of $13.2 \mu \mathrm{m}$ (median value), p.Arg254Trp (c.760C $>$ T), p.Ser274Leu (c.821C $>$ T), p.Pro305Leu (c.914C $>$ T), and p.Arg316Trp (c.946C >T) exhibited run lengths of $6.7 \mu \mathrm{m}, 2.3 \mu \mathrm{m}, 7.5 \mu \mathrm{m}$, and $5.1 \mu \mathrm{m}$, respectively (Figure 5D).

\section{Discussion}

We describe the largest number of KAND individuals to date, including 62 missense variants, many of which were observed in single individuals. This suggests that we have not yet observed the full allelic spectrum in KAND. KIF1A is highly conserved. KIF1A has both fewer loss-of-function variants (observed/expected $=0.07$ ) and fewer missense variants than would be expected (0.57) ${ }^{59}$ We observed both dominant and recessive inheritance patterns, with the majority of KAND individuals seen with heterozygous missense variants. Predicted loss-of-function variants were associated with milder disease than missense alleles.

In the literature, the majority of individuals diagnosed with KIF1A-associated HSAN had biallelic loss-of-function variants in the alternatively spliced exon $25 \mathrm{~b}$, though one individual had, in trans, one loss-of-function variant in exon $25 \mathrm{~b}$ and a second loss-offunction in exon $45 .^{2}$ In all families with recessive KIF1A-associated HSAN, individuals heterozygous for a single loss-of-function variant were asymptomatic. There were notable differences between the individuals with KIF1A-associated HSAN who had homozygous variants in exon $25 \mathrm{~b}$ and the single individual who was heterozygous for variants in exons $25 \mathrm{~b}$ and 45 . All the homozygous individuals had disease onset in early adolescence (mean: 9.7 years; SD: 3.0 years), in contrast with the congenital disease seen in the compound 
heterozygous individual. Similarly, none of the homozygous individuals had any reported cognitive issues or issues with gait, while the compound heterozygous individual had delayed speech development with poor articulation and an IQ of 80 as well as short stature and difficulties with gait/ambulation requiring use of a wheelchair. ${ }^{2}$ However, there was only one individual with HSAN who had a loss-of-function allele outside of exon 25b, so replication is required.

Heterozygous loss-of-function variants reported in the literature including both premature termination and frameshift variants in KIF1A were associated with HSP. These individuals, some in their 60s at the time of report, all had normal cognition, normal MRIs, and no history of the visual problems or seizures common in our cohort. ${ }^{32}$ If we hypothesize these loss-of-function variants result in nonsense-mediated decay of the mutant transcript, this suggests that up to a $50 \%$ reduction in protein can potentially be tolerated with minimal clinical symptoms for many decades.

The clinical disease severity associated with missense variants in our study was much worse than any of the loss-of-function alleles, suggesting a dominant negative disease mechanism. Since KIF1A predominantly functions as a dimer, if the aberrant and normal proteins dimerize to a similar degree, heterozygous individuals should only have $25 \%$ of the dimeric protein that is normal. Variants resulting in decreased or no MT binding, like c.38G >A (p.Arg13His) and c.821C $>\mathrm{T}$ (p.Ser274Leu) (Figure S2), are associated with relatively milder disease, perhaps because aberrant protein homodimers are unable to bind to MTs and therefore cannot interfere globally with MT traffic but only lead to decreased transport of KIF1A cargo. In contrast, variants including c.751G > A (p.Gly251Arg) and c.757G>A (p.Glu253Lys), which result in tight binding to the MT but are unable to engage in processive motion, were associated with the most severe disease. These rigor proteins likely lead to the most severe impact on all traffic along MTs and may act as roadblocks, resulting in an overall deficiency of cargo transport along the associated MT. ${ }^{60}$ For alleles like p.Arg254Trp, p.Pro305Leu, and p.Arg316Trp, where the aberrant motors bind to MTs but show reduced processivities and velocities (Figures 5C and 5D), in vivo these kinesins may affect MT transport globally and could interfere with all traffic along MTs.

While a previous study suggested that motors generated with the c.760C $>\mathrm{T}$ (p.Arg254Trp) and c. $946 \mathrm{C}>\mathrm{T}$ (p.Arg316Trp) variants were incapable of binding to and moving along MTs, we found that these aberrant motors were processive (the published results on the p.Arg13His, p.Thr99Met, and p.Glu253Lys agree with our findings). ${ }^{57}$ The reason for this discrepancy is unclear.

With regard to many core features of the condition, our cohort overall is similar to previously described individuals from the literature (Table 1). The two main differences we see are a higher occurrence of hypotonia in our cohort and a lower occurrence of neuroimaging abnormalities. The cohort from the literature with sufficient data to directly compare with our cohort is older, with an average age of 14.7 years and a median of 9.5 years. Since the neuroimaging findings tend to develop over time, this could explain the difference in the frequency of neuroimaging abnormalities observed and may account for some of the difference in hypotonia. We include in our count both individuals in our cohort 
with current hypotonia and those with previous hypotonia that has resolved; if the individuals from the literature only reflected the current presence or absence of hypotonia in the modestly older cohort, this may contribute to the lower reported occurrence. Consistent with previous reports, the majority of disease-associated KIF1A variants we report are located in the protein's motor domain (Tables S4 and S5). Among the 62 disease-associated variants we describe in this study, currently 23 are pathogenic, 35 are likely pathogenic, and four are variants of uncertain clinical significance by ACMG criteria. Compared to the initial variant classification by the clinical lab at time of testing, the majority of variant classifications were unchanged, but six variants were upgraded from likely pathogenic to pathogenic, two variants were upgraded from variant of uncertain significance to pathogenic, and one was upgraded from variant of unknown significance to likely pathogenic, while twelve were downgraded from pathogenic to likely pathogenic and one was downgraded from likely pathogenic to variant of uncertain significance. Among the downgraded variants, this was always because we did not consider the presence of a variant in the protein's motor domain as sufficient to meet ACMG criterion PM1, given the presence of many benign variants within the motor domain. The variants we upgraded were primarily individuals where multiple unrelated individuals were identified with the same phenotype. The four variants of uncertain clinical significance were observed in two unrelated individuals with recessive disease associated with inherited heterozygous variants in trans. In both individuals, the variants were identified through trio exome sequencing to be the most likely explanation for the individual's disease. Using our clinical severity score, we observed that the most severe phenotypes were associated with variants clustered near the ATP and MT binding sites (Figure 5A). This includes the p.Thr99Met, p.Gly251 Arg, and p.Glu253Lys variants, whose rigor phenotype likely represents defects in KIF1A's capacity to hydrolyze ATP. Our cohort was enriched for more severely affected individuals, but, in the literature, there were many individuals with missense variants in the motor domain with milder symptoms, more akin to pure HSP. It is likely these variants have an alternative mechanism of action, perhaps by hyperactivating motors, as was suggested in a recent study. ${ }^{61}$

One important limitation in the application of our disease severity score to understand something about the functional regions of the protein is that by virtue of the fact that KAND is a progressive condition, someone with a severity score in the mild range at 3 years of age may over the course of her life progress to a score in the moderate range as additional symptoms manifest over time. While age is a contributor to disease severity in any progressive condition, and we do see individuals with scores in the mild range on the younger side, the similarity in age distribution between individuals with scores in the moderate and severe ranges shows age is insufficient to explain the difference in severity. Given the differing disease mechanisms suggested by our functional data, we conclude this severity difference reflects fundamental differences in disease mechanism.

The relatively recent discovery that $K I F 1 A$ variants can cause disease as well as the changing clinical practice of how and when to incorporate genetic testing skew the age distribution of this study toward younger individuals, which makes it difficult to determine lifetime prevalence of these specific symptoms in the KAND population. We believe there are likely many older affected individuals who would be diagnosed with KAND if given 
appropriate genetic testing, and we hope identification of those individuals will help us better understand the clinical spectrum.

Beyond the genotype/phenotype correlations we observe when looking at variant severity in larger functional regions, we see some focal locations associated with very severe disease: p.Gly251Arg and p.Glu253Lys are two of the most severe variants we see, and they occur very close to each other. While we observed some phenotype/genotype correlations, the correlations are imperfect, but this is not surprising. There are likely other contributors to the clinical phenotype, including interacting genetic factors, prenatal and postnatal exposures, and the effects of therapeutic interventions. Our cohort included two sets of monozygotic twins, and in both instances, there were differences between the twins. In one family, one twin developed seizures in her teens, while her sister did not develop seizures until her twenties. Many of these clinical features developed over time, and individuals in our cohort ranged in age in our cross-sectional study. With sufficient longitudinal data, we will assess how the scores and the disease change over time. An objective biomarker of neurodegeneration might provide a useful tool in conjunction with the clinical severity score.

The allelic heterogeneity of this condition is striking. We observed a range of severity across inheritance patterns, variant types, and variant locations. To assist with clearer communication about KAND, we propose three KAND subtypes: simple KAND, complex KAND, and HSAN KAND. We suggest distinguishing between individuals with only progressive lower extremity spasticity, ataxia, and peripheral neuropathy (simple KAND) and individuals with developmental delay, intellectual disability, seizures, optic nerve atrophy, and cerebellar atrophy (complex KAND). Complex KAND would include all individuals previously diagnosed with MRD9/NASCAV, PEHO, or KIF1A-related Rett syndrome. HSAN KAND would include individuals with variants in the alternatively spliced exon described previously. This proposed classification system could inform clinical management, and we suggest that all individuals with complex KAND would benefit from increased surveillance to detect the presence of seizures and progressive vision loss.

This study expands our understanding of the allelic spectrum and range of clinical phenotypes in KAND and begins to address the molecular mechanisms of the disorder. With this broad phenotypic spectrum and the large number of variants, it is likely KAND is underrecognized and underdiagnosed. Better understanding of the phenotypic breadth and the disease subtypes could lead to improvements in diagnosis. As we move beyond diagnoses and work toward treatments, the ability to accurately predict prognosis and classify variants by molecular mechanism will facilitate the development of treatments.

\section{Supplementary Material}

Refer to Web version on PubMed Central for supplementary material.

\section{Acknowledgments}

We thank KIF1A.org for their ongoing partnership and Autism Brain Net for the collection of postmortem brain specimens. We thank Scott Robinson, Jennifer Shahar, Emily Horowitz, and Rachel Cogny for their assistance with this project. We thank Kristen Verhey for her assistance with optimizing the neurite tip accumulation and in vitro gliding assays. Molecular graphics were generated with UCSF Chimera, developed by the Resource for 
Biocomputing, Visualization, and Informatics at the University of California, San Francisco, with support from NIH grant P41-GM103311. Support was also received from NIH grants TL1TR001875 (L.B.), R01N114636 (L.R., A.G., and W.K.C.), and UL1TR001873 and from KIF1A.org. Research conducted at the Murdoch Children's Research Institute was supported by the Victorian Government's Operational Infrastructure Support Program.

\section{Data and code availability}

There are restrictions to the availability of the complete phenotypic data generated and analyzed during this study due to confidentiality concerns that individual study participants are identifiable from a complete data set. A large nearly complete data set is available from the corresponding author on request.

\section{References}

1. Alfadhel M, Yong SL, Lillquist Y, and Langlois S (2011). Precocious puberty in two girls with PEHO syndrome: a clinical feature not previously described. J. Child Neurol 26, 851-857. [PubMed: 21596701]

2. Rivière J-B, Ramalingam S, Lavastre V, Shekarabi M, Holbert S, Lafontaine J, Srour M, Merner N, Rochefort D, Hince P, et al. (2011). KIF1A, an axonal transporter of synaptic vesicles, is mutated in hereditary sensory and autonomic neuropathy type 2. Am. J. Hum. Genet 89, 219-230. [PubMed: 21820098]

3. Erlich Y, Edvardson S, Hodges E, Zenvirt S, Thekkat P, Shaag A, Dor T, Hannon GJ, and Elpeleg O (2011). Exome sequencing and disease-network analysis of a single family implicate a mutation in KIF1A in hereditary spastic paraparesis. Genome Res 21, 658-664. [PubMed: 21487076]

4. Hamdan FF, Gauthier J, Araki Y, Lin DT, Yoshizawa Y, Higashi K, Park AR, Spiegelman D, Dobrzeniecka S, Piton A, et al.; S2D Group (2011). Excess of de novo deleterious mutations in genes associated with glutamatergic systems in nonsyndromic intellectual disability. Am. J. Hum. Genet 88, 306-316. [PubMed: 21376300]

5. Klebe S, Lossos A, Azzedine H, Mundwiller E, Sheffer R, Gaussen M, Marelli C, Nawara M, Carpentier W, Meyer V, et al. (2012). KIF1A missense mutations in SPG30, an autosomal recessive spastic paraplegia: distinct phenotypes according to the nature of the mutations. Eur. J. Hum. Genet 20, 645-649. [PubMed: 22258533]

6. Okamoto N, Miya F, Tsunoda T, Yanagihara K, Kato M, Saitoh S, Yamasaki M, Kanemura Y, and Kosaki K (2014). KIF1A mutation in a patient with progressive neurodegeneration. J. Hum. Genet 59, 639-641. [PubMed: 25253658]

7. Jamuar SS, and Walsh CA (2014). Somatic mutations in cerebral cortical malformations. N. Engl. J. Med 371, 2038.

8. Lee J-R, Srour M, Kim D, Hamdan FF, Lim SH, Brunel-Guitton C, Décarie JC, Rossignol E, Mitchell GA, Schreiber A, et al. (2015). De novo mutations in the motor domain of KIF1A cause cognitive impairment, spastic paraparesis, axonal neuropathy, and cerebellar atrophy. Hum. Mutat 36, 69-78. [PubMed: 25265257]

9. Esmaeeli Nieh S, Madou MRZ, Sirajuddin M, Fregeau B, McKnight D, Lexa K, Strober J, Spaeth C, Hallinan BE, Smaoui N, et al. (2015). De novo mutations in KIF1A cause progressive encephalopathy and brain atrophy. Ann. Clin. Transl. Neurol 2, 623-635. [PubMed: 26125038]

10. Ylikallio E, Kim D, Isohanni P, Auranen M, Kim E, Lönnqvist T, and Tyynismaa H (2015). Dominant transmission of de novo KIF1A motor domain variant underlying pure spastic paraplegia. Eur. J. Hum. Genet 23, 1427-1430. [PubMed: 25585697]

11. Citterio A, Arnoldi A, Panzeri E, Merlini L, D’Angelo MG, Musumeci O, Toscano A, Bondi A, Martinuzzi A, Bresolin N, and Bassi MT (2015). Variants in KIF1A gene in dominant and sporadic forms of hereditary spastic paraparesis. J. Neurol 262, 2684-2690. [PubMed: 26410750]

12. Ohba C, Haginoya K, Osaka H, Kubota K, Ishiyama A, Hiraide T, Komaki H, Sasaki M, Miyatake S, Nakashima M, et al. (2015). De novo KIF1A mutations cause intellectual deficit, cerebellar atrophy, lower limb spasticity and visual disturbance. J. Hum. Genet 60, 739-742. [PubMed: 26354034] 
13. Megahed H, Nicouleau M, Barcia G, Medina-Cano D, Siquier-Pernet K, Bole-Feysot C, Parisot M, Masson C, Nitschké P, Rio M, et al. (2016). Utility of whole exome sequencing for the early diagnosis of pediatric-onset cerebellar atrophy associated with developmental delay in an inbred population. Orphanet J. Rare Dis 11, 57. [PubMed: 27146152]

14. Hotchkiss L, Donkervoort S, Leach ME, Mohassel P, Bharucha-Goebel DX, Bradley N, Nguyen D, Hu Y, Gurgel-Giannetti J, and Bönnemann CG (2016). Novel De Novo Mutations in KIF1A as a Cause of Hereditary Spastic Paraplegia With Progressive Central Nervous System Involvement. J. Child Neurol 31, 1114-1119. [PubMed: 27034427]

15. Iqbal Z, Rydning SL, Wedding IM, Koht J, Pihlstrøm L, Rengmark AH, Henriksen SP, Tallaksen CME, and Toft M (2017). Targeted high throughput sequencing in hereditary ataxia and spastic paraplegia. PloS One 12, e0174667. [PubMed: 28362824]

16. Hasegawa A, Koike R, Koh K, Kawakami A, Hara N, Takiyama Y, and Ikeuchi T (2017). Coexistence of spastic paraplegia-30 with novel KIF1A mutation and spinocerebellar ataxia 31 with intronic expansion of BEAN and TK2 in a family. J. Neurol. Sci 372, 128-130. [PubMed: 28017198]

17. Krenn M, Zulehner G, Hotzy C, Rath J, Stogmann E, Wagner M, Haack TB, Strom TM, Zimprich A, and Zimprich F (2017). Hereditary spastic paraplegia caused by compound heterozygous mutations outside the motor domain of the KIF1A gene. Eur. J. Neurol 24, 741-747. [PubMed: 28332297]

18. Cheon CK, Lim S-H, Kim Y-M, Kim D, Lee NY, Yoon TS, Kim NS, Kim E, and Lee JR (2017). Autosomal dominant transmission of complicated hereditary spastic paraplegia due to a dominant negative mutation of KIF1A, SPG30 gene. Sci. Rep 7, 12527. [PubMed: 28970574]

19. Roda RH, Schindler AB, and Blackstone C (2017). Multi-generation family with dominant SPG30 hereditary spastic paraplegia. Ann. Clin. Transl. Neurol 4, 821-824. [PubMed: 29159194]

20. Travaglini L, Aiello C, Stregapede F, D’Amico A, Alesi V, Ciolfi A, Bruselles A, Catteruccia M, Pizzi S, Zanni G, et al. (2018). The impact of next-generation sequencing on the diagnosis of pediatric-onset hereditary spastic paraplegias: new genotype-phenotype correlations for rare HSPrelated genes. Neurogenetics 19, 111-121. [PubMed: 29691679]

21. Demily C, Lesca G, Poisson A, Till M, Barcia G, Chatron N, Sanlaville D, and Munnich A (2018). Additive Effect of Variably Penetrant 22q11.2 Duplication and Pathogenic Mutations in Autism Spectrum Disorder: To Which Extent Does the Tree Hide the Forest? J. Autism Dev. Disord 48, 2886-2889. [PubMed: 29589274]

22. Lu C, Li L-X, Dong H-L, Wei Q, Liu ZJ, Ni W, Gitler AD, and Wu ZY (2018). Targeted nextgeneration sequencing improves diagnosis of hereditary spastic paraplegia in Chinese patients. J. Mol. Med. (Berl.) 96, 701-712. [PubMed: 29934652]

23. Samanta D, and Gokden M (2019). PEHO syndrome: KIF1A mutation and decreased activity of mitochondrial respiratory chain complex. J. Clin. Neurosci 61, 298-301. [PubMed: 30385166]

24. Tomaselli PJ, Rossor AM, Horga A, Laura M, Blake JC, Houlden H, and Reilly MM (2017). A de novo dominant mutation in KIF1A associated with axonal neuropathy, spasticity and autism spectrum disorder. J. Peripher. Nerv. Syst 22, 460-463. [PubMed: 28834584]

25. Yoshikawa K, Kuwahara M, Saigoh K, Ishiura H, Yamagishi Y, Hamano Y, Samukawa M, Suzuki H, Hirano M, Mitsui Y, et al. (2018). The novel de novo mutation of KIF1A gene as the cause for Spastic paraplegia 30 in a Japanese case. eNeurologicalSci 14, 34-37. [PubMed: 30582020]

26. Volk A, Conboy E, Wical B, Patterson M, and Kirmani S (2015). Whole-Exome Sequencing in the Clinic: Lessons from Six Consecutive Cases from the Clinician's Perspective. Mol. Syndromol 6, 23-31. [PubMed: 25852444]

27. Elert-Dobkowska E, Stepniak I, Krysa W, Ziora-Jakutowicz K, Rakowicz M, Sobanska A, Pilch J, Antczak-Marach D, Zaremba J, and Sulek A (2019). Next-generation sequencing study reveals the broader variant spectrum of hereditary spastic paraplegia and related phenotypes. Neurogenetics 20, 27-38. [PubMed: 30778698]

28. Sun M, Johnson AK, Nelakuditi V, Guidugli L, Fischer D, Arndt K, Ma L, Sandford E, Shakkottai V, Boycott K, et al. (2019). Targeted exome analysis identifies the genetic basis of disease in over $50 \%$ of patients with a wide range of ataxia-related phenotypes. Genet. Med 21, 195-206. [PubMed: 29915382] 
29. Muir AM, Myers CT, Nguyen NT, Saykally J, Craiu D, De Jonghe P, Helbig I, Hoffman-Zacharska D, Guerrini R, Lehesjoki AE, et al.; EuroEPINOMICS-RES NLES working group, Sarah Weckhuysen (2019). Genetic heterogeneity in infantile spasms. Epilepsy Res 156, 106181. [PubMed: 31394400]

30. Kashimada A, Hasegawa S, Nomura T, Shiraku H, Moriyama K, Suzuki T, Nakajima K, Mizuno T, Imai K, Sugawara Y, et al. (2019). Genetic analysis of undiagnosed ataxia-telangiectasia-like disorders. Brain Dev 41, 150-157. [PubMed: 30301590]

31. Jacob D, Peter T, and Nick G (2018). A novel Kif1a mutation causing a neurodevelopmental disorder. J Neurol Neurosurg Psychiatry 89, A29.

32. Pennings M, Schouten MI, van Gaalen J, Meijer RPP, de Bot ST, Kriek M, Saris CGJ, van den Berg LH, van Es MA, Zuidgeest DMH, et al. (2020). KIF1A variants are a frequent cause of autosomal dominant hereditary spastic paraplegia. Eur. J. Hum. Genet 28, 40-49. [PubMed: 31488895]

33. Xiaojing W, Yanyan M, Ruonan D, Xiaolin L, Haiyan Z, Jian M, Yi L, Wenjie S, and Qiji L (2020). Generation of a human induced pluripotent stem cell line (SDUBMSi001-A) from a hereditary spastic paraplegia patient carrying kifla c.773C $>$ T missense mutation. Stem Cell Res. (Amst.) 43, 101727.

34. van de Warrenburg BP, Schouten MI, de Bot ST, Vermeer S, Meijer R, Pennings M, Gilissen C, Willemsen MA, Scheffer H, and Kamsteeg EJ (2016). Clinical exome sequencing for cerebellar ataxia and spastic paraplegia uncovers novel gene-disease associations and unanticipated rare disorders. Eur. J. Hum. Genet 24, 1460-1466. [PubMed: 27165006]

35. Kaur S, Van Bergen NJ, Verhey KJ, Nowell CJ, Budaitis B, Yue Y, Ellaway C, Brunetti-Pierri N, Cappuccio G, Bruno I, et al. (2020). Expansion of the phenotypic spectrum of de novo missense variants in kinesin family member 1A (KIF1A). Hum. Mutat 41, 1761-1774.

36. Raffa L, Matton M-P, Michaud J, Rossignol E, Décarie J-C, and Ospina LH (2017). Optic nerve hypoplasia in a patient with a de novo KIF1A heterozygous mutation. Can. J. Ophthalmol 52, e169-e171. [PubMed: 28985824]

37. Hosokawa S, Kubo Y, Arakawa R, Takashima H, and Saito K (2020). Analysis of spinal muscular atrophy-like patients by targeted resequencing. Brain Dev 42, 148-156. [PubMed: 31734026]

38. Spagnoli C, Rizzi S, Salerno GG, Frattini D, and Fusco C (2019). Long-term follow-up until early adulthood in autosomal dominant, complex SPG30 with a novel KIF1A variant: a case report. Ital. J. Pediatr 45, 155. [PubMed: 31796088]

39. Ishiura H, Bannai T, Mitsui J, Yoshimura J, Morishita S, Hayashi T, Shimizu J, Toda T, and Tsuji S (2020). A Novel de novo KIF1A Mutation in a Patient with Autism, Hyperactivity, Epilepsy, Sensory Disturbance, and Spastic Paraplegia. Intern. Med 59, 839-842. [PubMed: 31813911]

40. Wang J, Zhang Q, Chen Y, Yu S, Wu X, and Bao X (2019). Rett and Rett-like syndrome: Expanding the genetic spectrum to KIF1A and GRIN1 gene. Mol. Genet. Genomic Med 7, e968. [PubMed: 31512412]

41. Nemani T, Steel D, Kaliakatsos M, DeVile C, Ververi A, Scott R, Getov S, Sudhakar S, Male A, Mankad K, et al.; Genomics England Research Consortium (2020). KIF1A-related disorders in children: A wide spectrum of central and peripheral nervous system involvement. J. Peripher. Nerv. Syst 25, 117-124. [PubMed: 32096284]

42. Guo Y, Chen Y, Yang M, Xu X, Lin Z, Ma J, Chen H, Hu Y, Ma Y, Wang X, and Tian X (2020). A Rare KIF1A Missense Mutation Enhances Synaptic Function and Increases Seizure Activity. Front. Genet 11, 61. [PubMed: 32174959]

43. Langlois S, Tarailo-Graovac M, Sayson B, Drögemöller B, Swenerton A, Ross CJ, Wasserman WW, and van Karnebeek CD (2016). De novo dominant variants affecting the motor domain of KIF1A are a cause of PEHO syndrome. Eur. J. Hum. Genet 24, 949-953. [PubMed: 26486474]

44. Hirokawa N, Nitta R, and Okada Y (2009). The mechanisms of kinesin motor motility: lessons from the monomeric motor KIF1A. Nat. Rev. Mol. Cell Biol 10, 877-884. [PubMed: 19935670]

45. Tanaka Y, Niwa S, Dong M, Farkhondeh A, Wang L, Zhou R, and Hirokawa N (2016). The Molecular Motor KIF1A Transports the TrkA Neurotrophin Receptor and Is Essential for Sensory Neuron Survival and Function. Neuron 90, 1215-1229. [PubMed: 27263974] 
46. Richards S, Aziz N, Bale S, Bick D, Das S, Gastier-Foster J, Grody WW, Hegde M, Lyon E, Spector E, et al. (2015). Standards and guidelines for the interpretation of sequence variants: a joint consensus recommendation of the American College of Medical Genetics and Genomics and the Association for Molecular Pathology. Genet. Med 17, 405-424. [PubMed: 25741868]

47. Lek M, Karczewski KJ, Minikel EV, Samocha KE, Banks E, Fennell T, O’Donnell-Luria AH, Ware JS, Hill AJ, Cummings BB, et al.; Exome Aggregation Consortium (2016). Analysis of proteincoding genetic variation in 60,706 humans. Nature 536, 285-291. [PubMed: 27535533]

48. Auton A, Brooks LD, Durbin RM, Garrison EP, Kang HM, Korbel JO, Marchini JL, McCarthy S, McVean GA, and Abecasis GR; 1000 Genomes Project Consortium (2015). A global reference for human genetic variation. Nature 526, 68-74. [PubMed: 26432245]

49. Monroy BY, Sawyer DL, Ackermann BE, Borden MM, Tan TC, and Ori-McKenney KM (2018). Competition between microtubule-associated proteins directs motor transport. Nat. Commun 9, 1487. [PubMed: 29662074]

50. Hammond JW, Cai D, Blasius TL, Li Z, Jiang Y, Jih GT, Meyhofer E, and Verhey KJ (2009). Mammalian Kinesin-3 motors are dimeric in vivo and move by processive motility upon release of autoinhibition. PLoS Biol 7, e72. [PubMed: 19338388]

51. Rao L, Berger F, Nicholas MP, and Gennerich A (2019). Molecular mechanism of cytoplasmic dynein tension sensing. Nat. Commun 10, 3332. [PubMed: 31350388]

52. Nicholas MP, Rao L, and Gennerich A (2014). An improved optical tweezers assay for measuring the force generation of single kinesin molecules. Methods Mol. Biol 1136, 171-246. [PubMed: 24633799]

53. Atherton J, Farabella I, Yu IM, Rosenfeld SS, Houdusse A, Topf M, and Moores CA (2014). Conserved mechanisms of microtubule-stimulated ADP release, ATP binding, and force generation in transport kinesins. Elife 3, e03680. [PubMed: 25209998]

54. Kikkawa M, and Hirokawa N (2006). High-resolution cryo-EM maps show the nucleotide binding pocket of KIF1A in open and closed conformations. EMBO J 25, 4187-4194. [PubMed: 16946706]

55. Budaitis BG, Jariwala S, Rao L, Sept D, Verhey KJ, and Gennerich A (2021). Pathogenic Mutations in the Kinesin-3 Motor KIF1A Diminish Force Generation and Movement Through Allosteric Mechanisms. J. Cell Biol 220, e202004227. [PubMed: 33496723]

56. Soppina V, and Verhey KJ (2014). The family-specific K-loop influences the microtubule on-rate but not the superprocessivity of kinesin-3 motors. Mol Biol. Cell 25, 2161-2170. [PubMed: 24850887]

57. Guedes-Dias P, Nirschl JJ, Abreu N, Tokito MK, Janke C, Magiera MM, and Holzbaur ELF (2019). Kinesin-3 Responds to Local Microtubule Dynamics to Target Synaptic Cargo Delivery to the Presynapse. Curr. Biol 29, 268-282.e8. [PubMed: 30612907]

58. Rao L, Hülsemann M, and Gennerich A (2018). Combining Structure-Function and SingleMolecule Studies on Cytoplasmic Dynein. Methods Mol. Biol 1665, 53-89. [PubMed: 28940064]

59. Karczewski KJ, Francioli LC, Tiao G, Cummings BB, Alföldi J, Wang Q, Collins RL, Laricchia KM, Ganna A, Birnbaum DP, et al. (2019). Variation across 141,456 human exomes and genomes reveals the spectrum of loss-of-function intolerance across human protein-coding genes. bioRxiv $10.1101 / 531210$.

60. Gabrych DR, Lau VZ, Niwa S, and Silverman MA (2019). Going Too Far Is the Same as Falling Shorty: Kinesin-3 Family Members in Hereditary Spastic Paraplegia. Front. Cell. Neurosci 13, 419. [PubMed: 31616253]

61. Chiba K, Takahashi H, Chen M, Obinata H, Arai S, Hashimoto K, Oda T, McKenney RJ, and Niwa S (2019). Disease-associated mutations hyperactivate KIF1A motility and anterograde axonal transport of synaptic vesicle precursors. Proc. Natl. Acad. Sci. USA 116, 18429-18434. [PubMed: 31455732] 

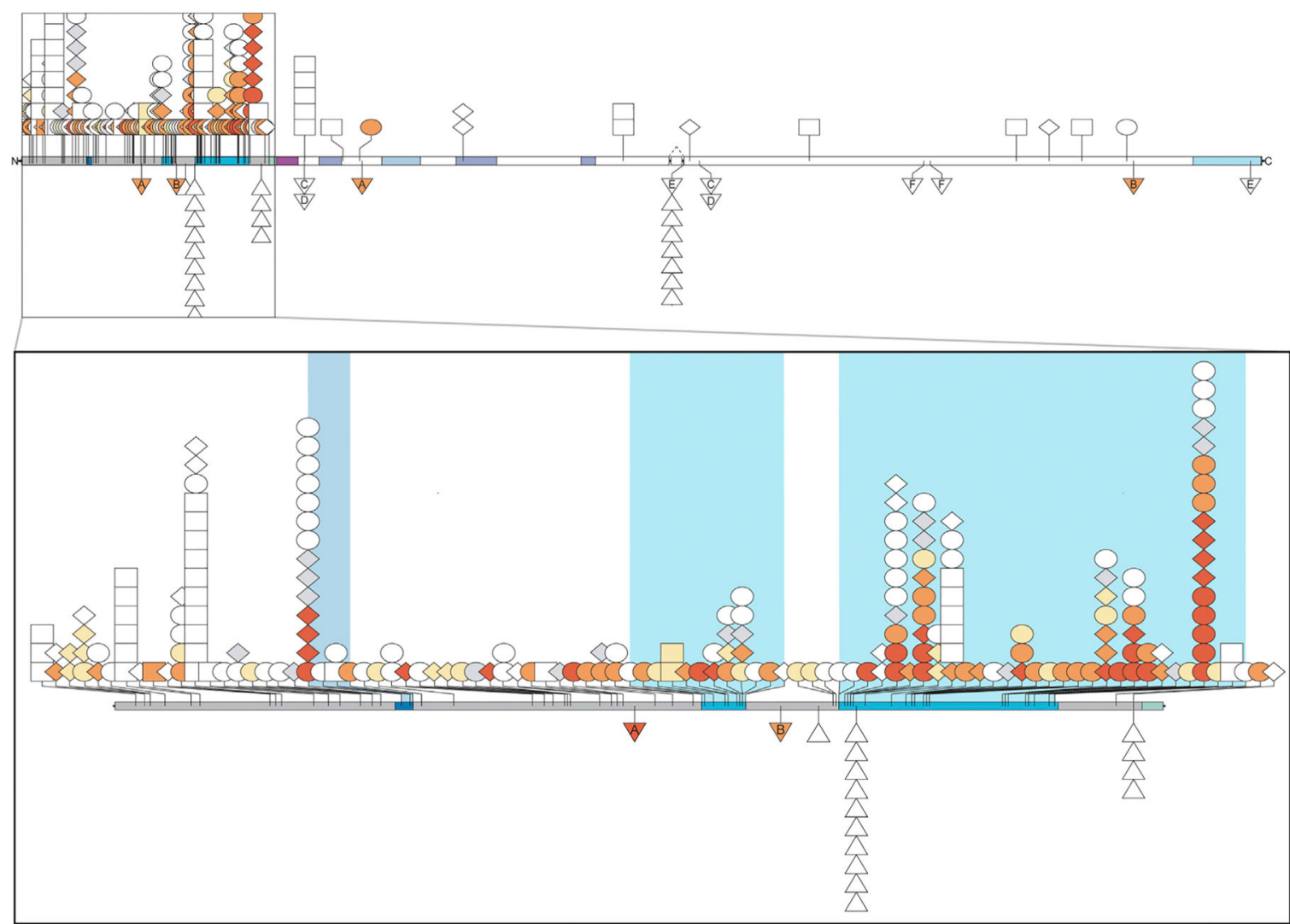

Variant key

Disease severity

$\diamond$ Mild $\diamond$ Moderate $\diamond$ Severe $\diamond$ Unknown $\diamond$ Literature

Inheritance

De novo $\diamond$ Dominant, inheritance unknown $\square$ Dominant inherited $\nabla$ Recessive, heterozygous $\triangle$ Recessive, homozygous

Structure key

ATP \& MT-binding regions

P-loop $\square$ Switch I $\square$ Switch II

Protein domains

$\square$ Motor $\square$ Neck coil $\square$ Coiled-coil $\square$ Forkhead associated $\square$ Plextrin homology

Figure 1. KIF1A variants by severity

Shapes representing 251 affected individuals are shown below, including 114 individuals

from our cohort and 137 individuals previously reported in the literature. Disease severity is shown for 98 of these individuals from our cohort. Four affected individuals are not incorporated, as their variants are not mappable below; this includes 3 variants affecting splicing ( 2 in our cohort, 1 in the literature) and one larger deletion from the literature. On top is the full-length protein, and on the bottom is a zoom in of the motor domain. Each icon represents a unique individual. The shape indicates inheritance and color indicates disease severity. In both the full-length protein and the motor domain, monoallelic variants are shown above and biallelic variants are shown below. Compound heterozygous individuals are represented by two separate triangles, one at each variant; those triangles have letters to indicate variant pairing. ATP and MT binding regions in the motor domain are indicated by color. Splicing information is only indicated for the alternatively spliced exon $25 \mathrm{~b}$. 


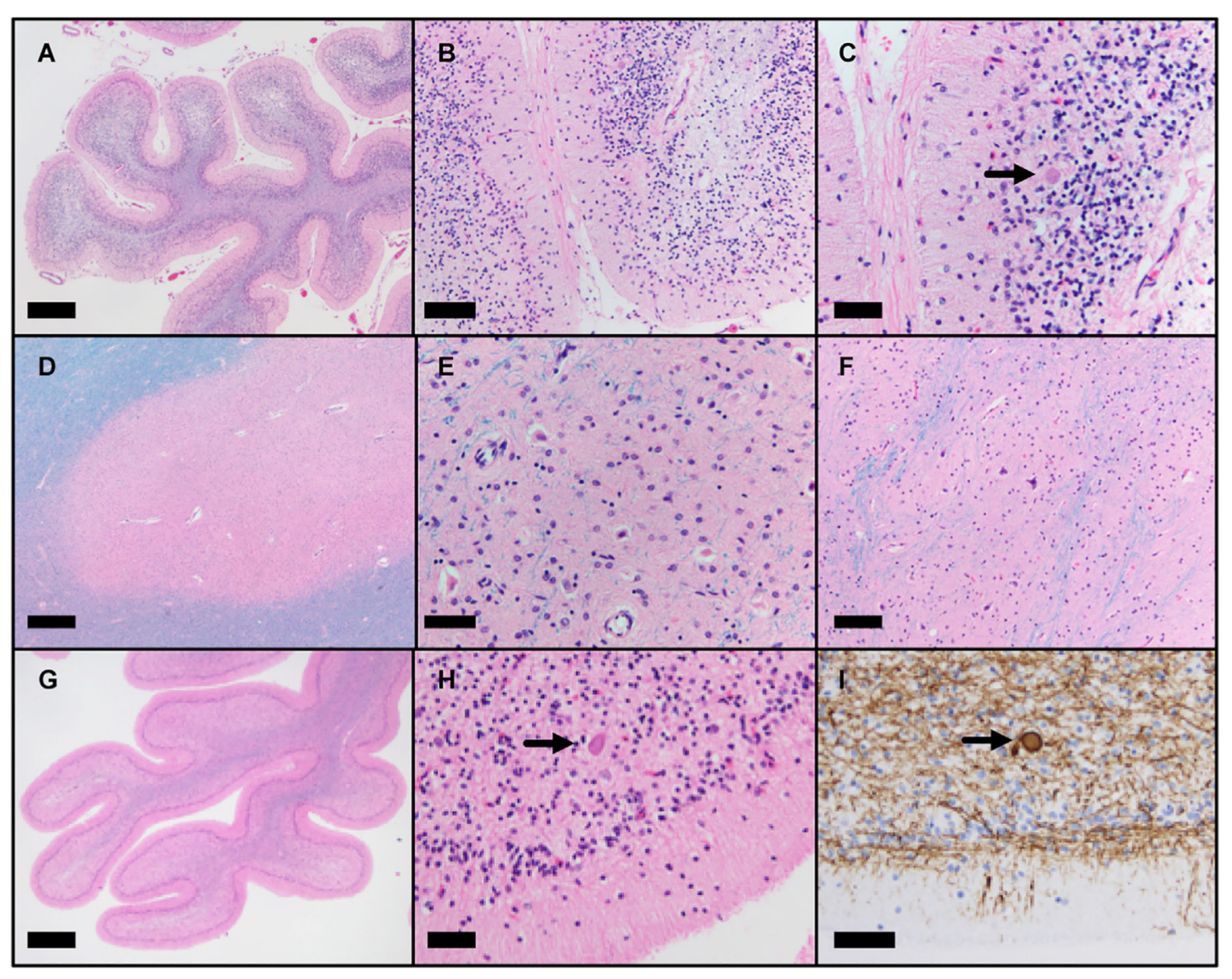

Figure 2. Neuropathological changes in two individuals with c.296C>T (p.Thr99Met) KIF1A variant

Microscopic findings from a 13-year-old female (A-F) and a 6-year-old female (G-I).

Microscopic examination of H\&E/Luxol fast blue-stained tissue sections reveal severely atrophic cerebellar folia (A and $\mathrm{G}$ ) with thinned molecular layer, depletion of Purkinje cells, depletion of internal granule cells, Bergmann gliosis, and pallor of the white matter (B, C, and $\mathrm{H}$ ). These changes are most severe in the superior aspect of the cerebellar vermis but are present in varying degrees throughout the cerebellum. Rare axonal spheroids are present (C and $\mathrm{H}$, arrow) highlighted by neurofilament immunostain (I). The dentate nucleus (D and E) as well as the inferior olivary nucleus of the medulla $(F)$ show marked loss of neurons and severe reactive gliosis. Residual neurons in these areas are hypereosinophilic with pyknotic nuclei. Scale bars: $1 \mathrm{~mm}(20 \times$ magnification) (A, D, and G), $200 \mu \mathrm{m}$ (100× magnification) (B and F), $100 \mu \mathrm{m}(200 \times$ magnification) $(\mathrm{C}, \mathrm{E}, \mathrm{H}$, and I). 
A

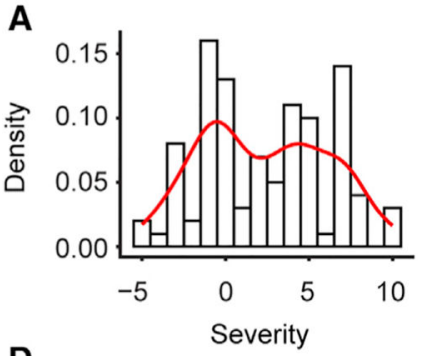

D

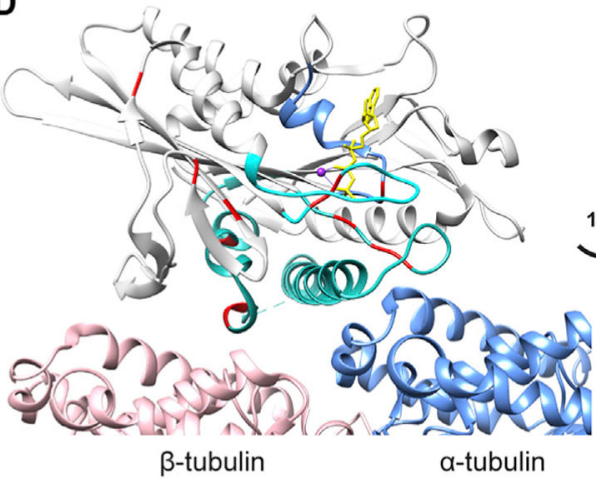

B

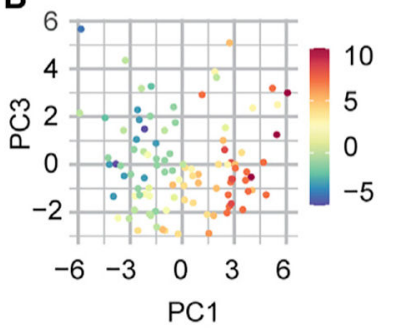

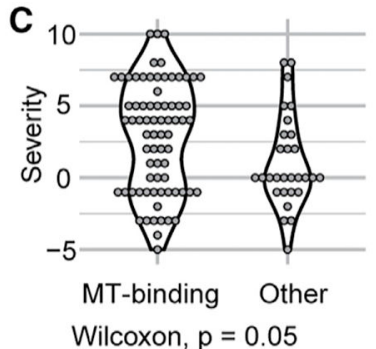

Wilcoxon, $p=0.05$

Figure 3. Clinical severity of $100 \mathrm{KAND}$ individuals

(A) Distribution of heuristic severity score.

(B) Scatterplot of principle components 1 and 3 color-coded by medical history-derived severity scores.

(C) Violin plot of medical history-derived severity scores for individuals with variants in or outside of ATP or MT binding regions.

(D) Structure of the MT-bound KIF1A motor domain with severe residues and functional regions involved in ATP and MT binding highlighted (PDB: 4UXP, H. sapiens KIF1A CryoEM structure in the presence of AMP-PNP). Light gray: KIF1A; blue: a-tubulin; pink: $\beta$-tubulin; yellow: AMP-PNP; red: residues mutated in most severe variants (p.Thr99, p.Glu148, p.Leu157, p.Va1186, p.Ser214, p.Ser215, p.Gly251, p.Glu253, p.Arg254, p.Tyr306, p.Arg307, p.Arg316). The P loop, switch I, and switch II are shown in light blue, cyan, and teal, respectively. 


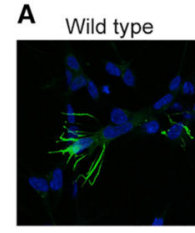

p.Ser217Tyr

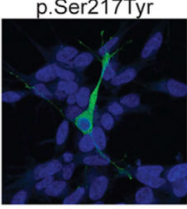

p.Arg254Pro

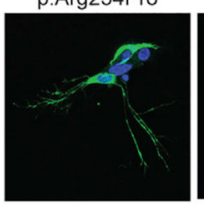

p. Thr344Met

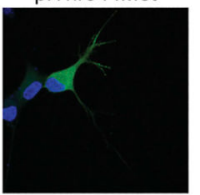

B

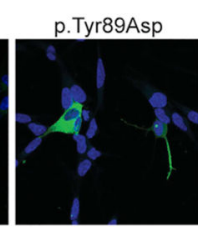

p.Asp248Gly

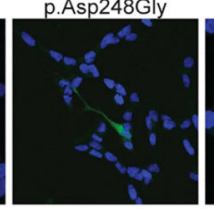

p.Leu278Pro
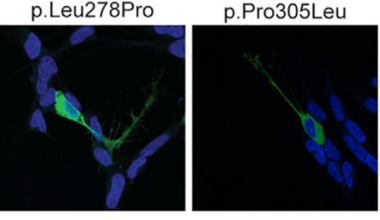

8.0
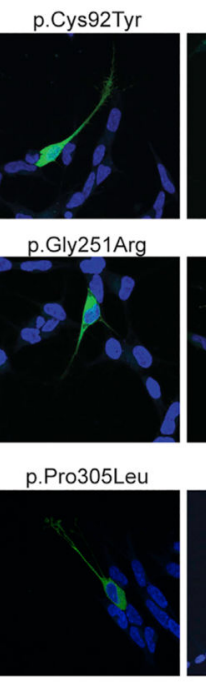

p.Gly251Arg

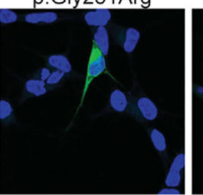

p.Arg254Trp
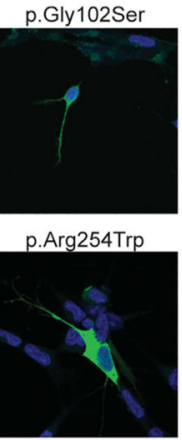

p.Arg307Pro
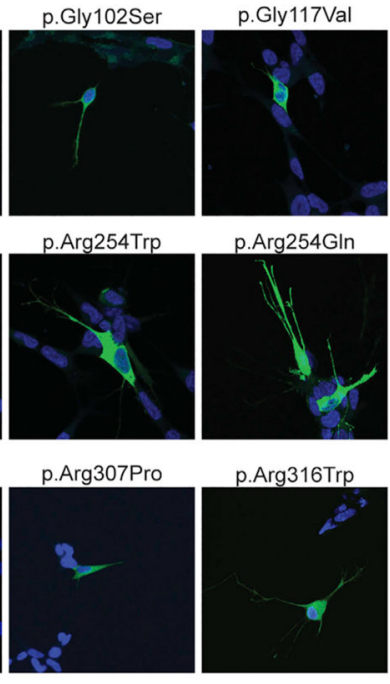

p.Arg254GIn

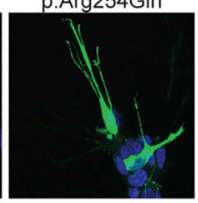

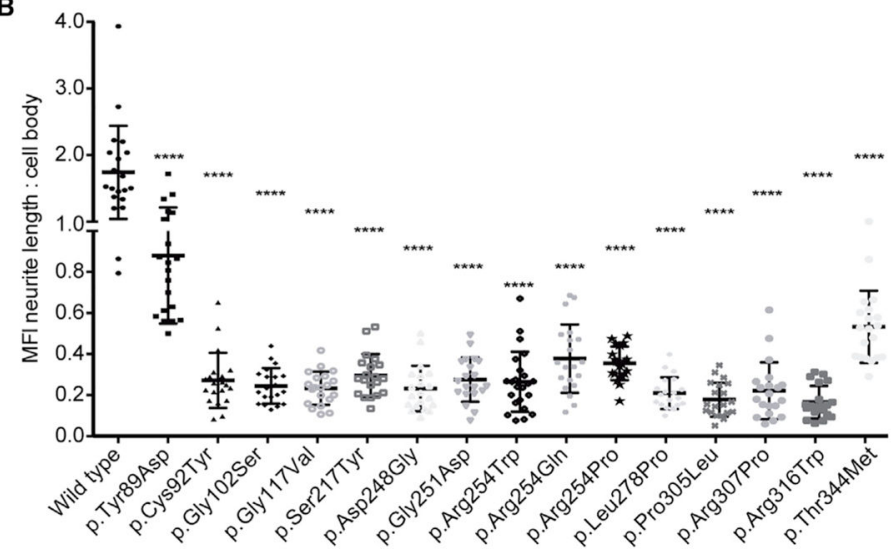

Figure 4. Neurite tip accumulation in differentiated SH-SY5Y cells for KIF1A variants seen in KAND individuals

(A) Rat KIF1A (1-393) tagged with 3xmCit tag (green) accumulated in the distal tips of differentiated SH-SY5Y cells in case of WT but was localized closer to the cell body in cells transfected with KIF1A constructs containing individual variants.

(B) A significant reduction in the mean florescence intensity (MFI) along the neurite length versus the cell body was observed in the 15 individual variants tested. Data were analyzed from three independent experiments using a Mann-Whitney test $(n=2)$ for each experiment) and are plotted as mean \pm SD. $* * * * p<0.0001$ versus WT. 
A
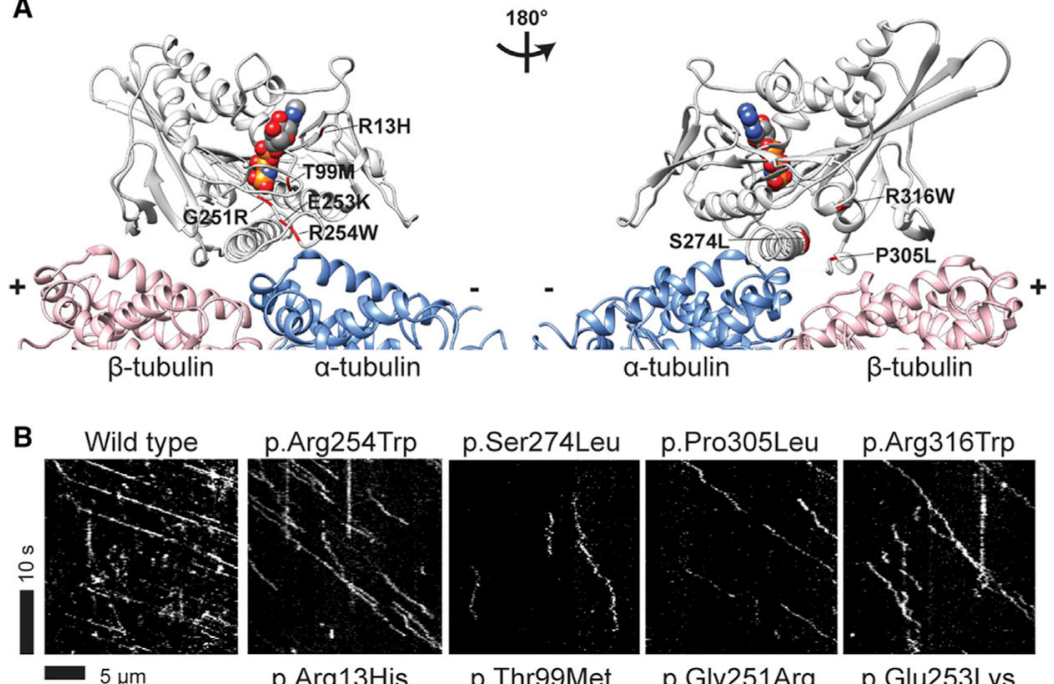

p.Ser274Leu

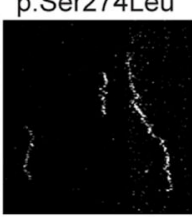

p.Arg13His

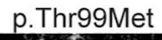

p.Thr99Met

p.Pro305Leu

p.Arg316Trp
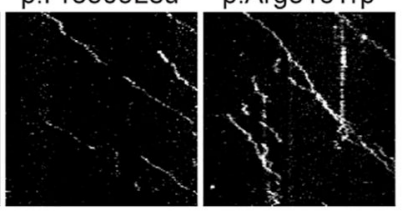

p.Gly251Arg

p.Glu253Lys
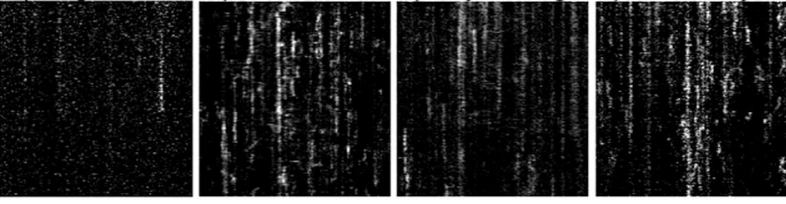

C
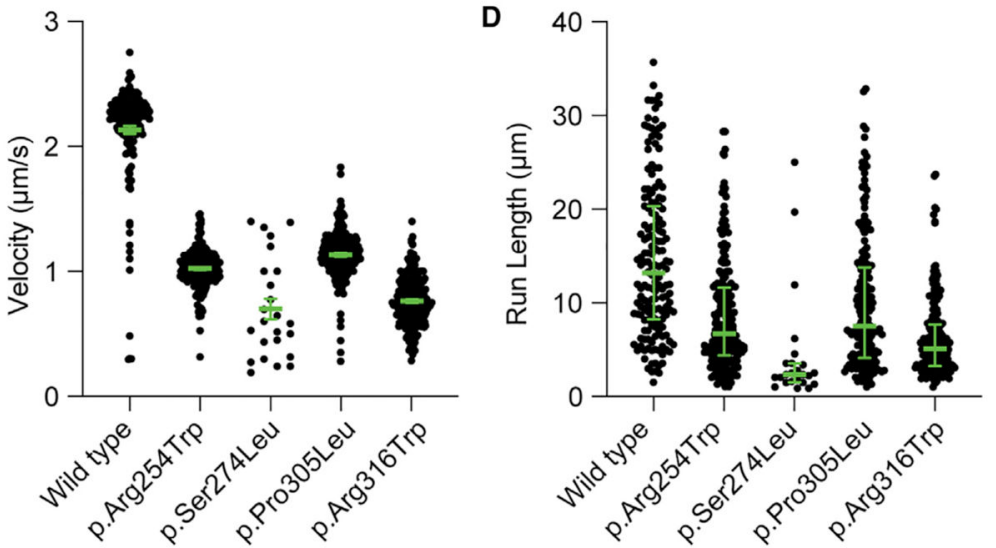

Figure 5. Sites of KIF1A mutations and motility analyses of dimeric KIF1A motors

(A) Structure of the MT-bound KIF1A motor domain with the sites of mutated residues used for TIRF microscopy. Light gray, KIF1A; blue, a-tubulin; pink, $\beta$-tubulin. Van der Walls sphere represents AMP-PNP; red residues represent the mutation site (PDB: 4UXP, $H$. sapiens KIF1A CryoEM structure in the presence of AMP-PNP). p.Arg13His, p.Thr99Met, p.Gly251Arg, p.Glu253Lys, and p.Arg254Trp are at or close to the ATP binding site of KIF1A (left). p.Ser274Leu, p.Pro305Leu, and p.Arg316Trp are close to the $\beta$-tubulin binding interface (right).

(B) Kymographs of KIF1A WT and mobile (top) and non-motile (bottom) motors. Diagonal lines in the kymograph represent KIF1A molecules that are moving over time. The depicted scale bars are the same for all kymographs shown in this figure.

(C) Velocities of KIF1A WT and the mobile motors. The green bars indicate the mean with SEM. Compared to WT, all the aberrant motors have reduced velocities. The WT has an 
average velocity of $2.1 \pm 0.03 \mu \mathrm{m} / \mathrm{s}($ mean \pm SEM) $(\mathrm{n}=162)$, while $\mathrm{p}$.Arg254Trp, p.Ser274Leu, p.Pro305Leu, and p.Arg316Trp move at $1.0 \pm 0.01 \mu \mathrm{m} / \mathrm{s}(\mathrm{n}=214), 0.7 \pm 0.08$ $\mu \mathrm{m} / \mathrm{s}(\mathrm{n}=24), 1.1 \pm 0.01 \mu \mathrm{m} / \mathrm{s}(\mathrm{n}=181)$, and $0.8 \pm 0.01 \mu \mathrm{m} / \mathrm{s}(\mathrm{n}=210)$, respectively. (D) Run lengths of KIF1A WT and aberrant motors. The green bars indicate the median with quartile. All the aberrant motors have reduced run lengths. The WT's processivity has a median value of $13.2(8.3,20.4) \mu \mathrm{m}$, while p.Arg254Trp, p.Ser274Leu, p.Pro305Leu, and p.Arg316Trp have run lengths of $6.7(4.4,11.6) \mu \mathrm{m}, 2.3(1.5,3.5) \mu \mathrm{m}, 7.5(4.1,13.8) \mu \mathrm{m}$, and $5.1(3.2,7.7) \mu \mathrm{m}$, respectively. 

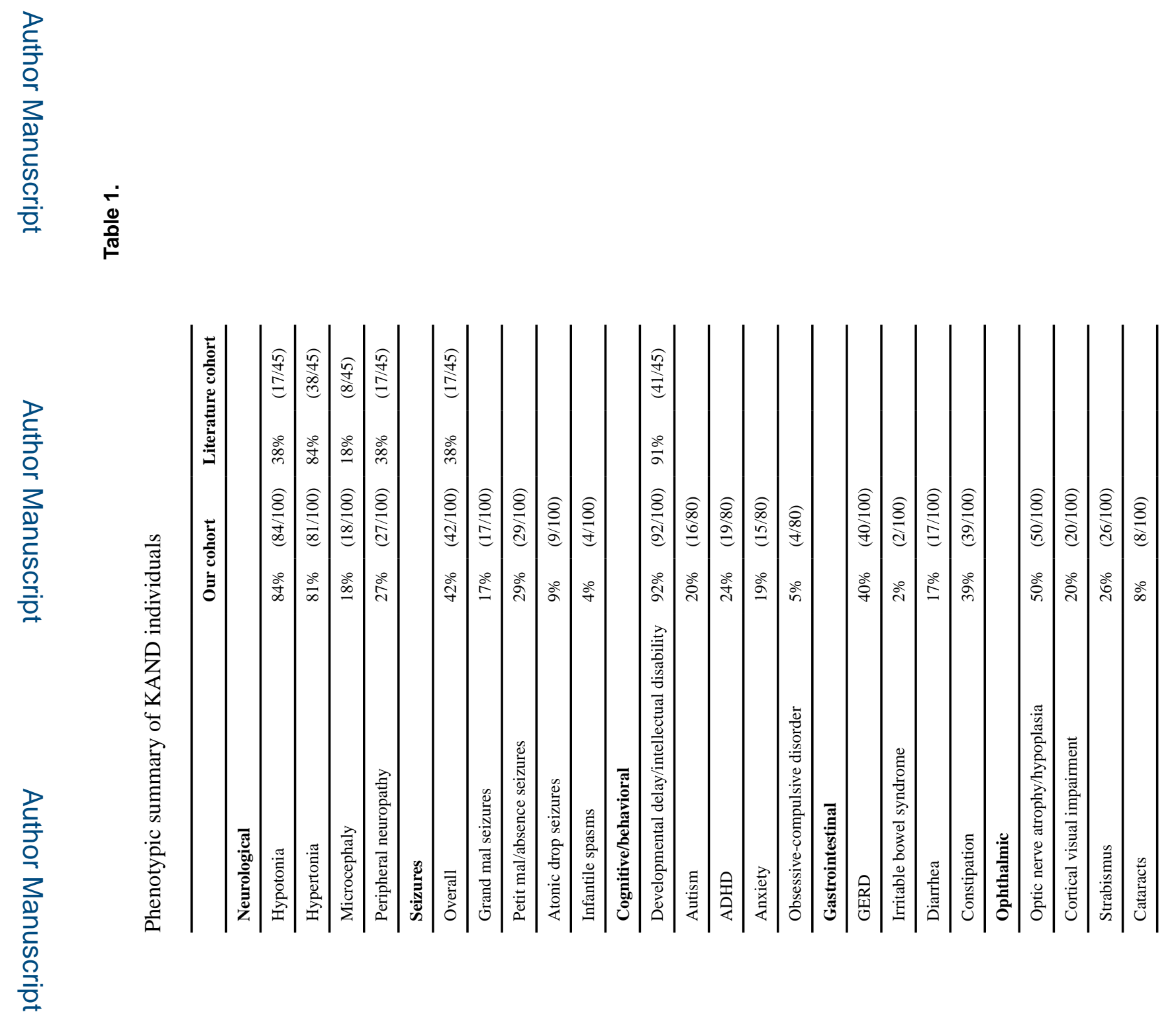

$H G G$ Adv. Author manuscript; available in PMC 2021 April 19. 


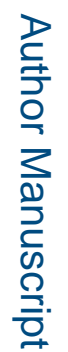

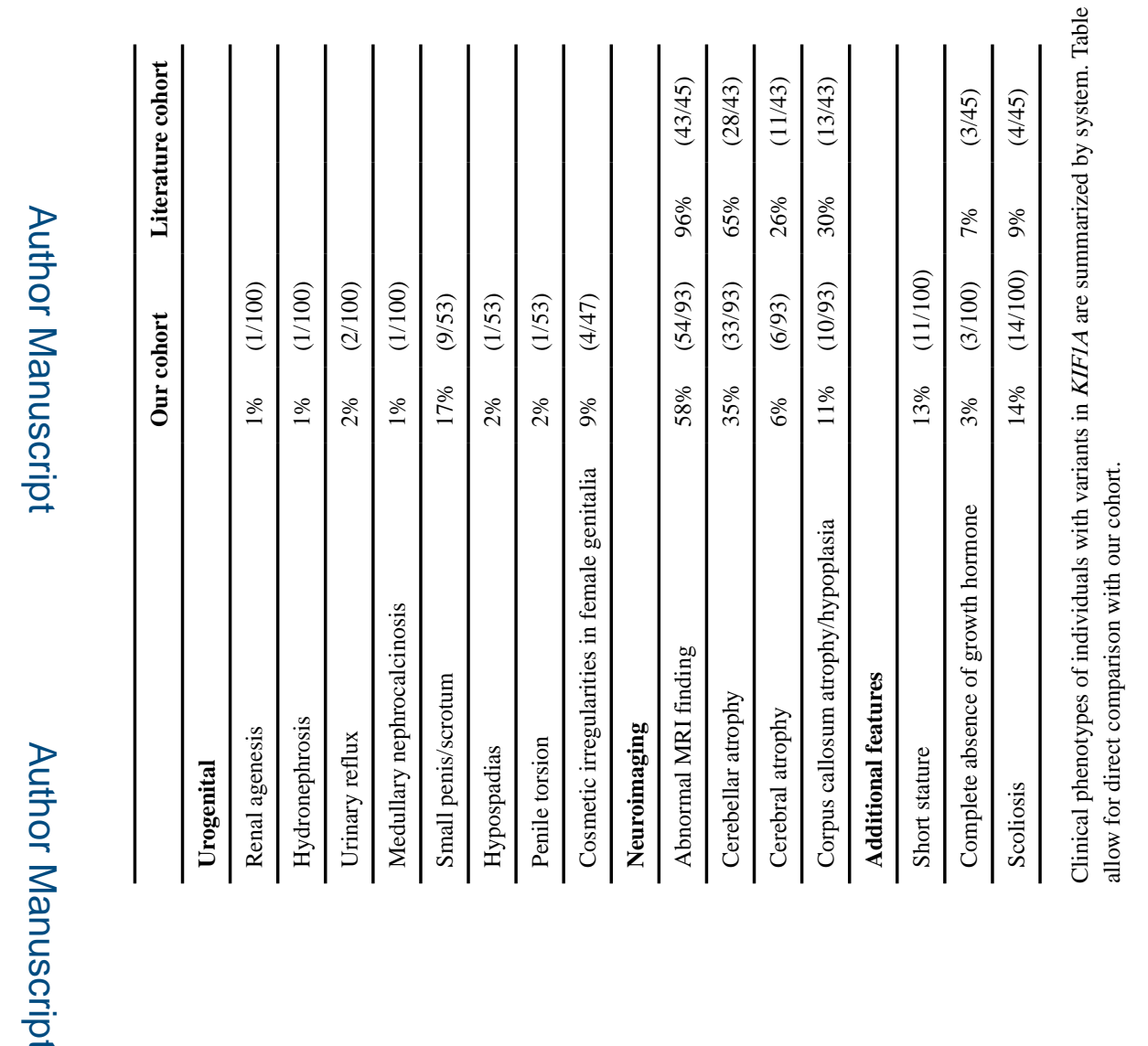

$H G G$ Adv. Author manuscript; available in PMC 2021 April 19. 


\section{University Library}

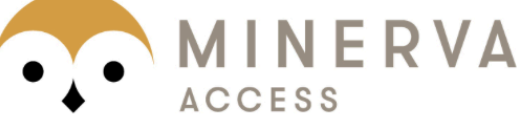

A gateway to Melbourne's research publications

Minerva Access is the Institutional Repository of The University of Melbourne

Author/s:

Boyle, L;Rao, L;Kaur, S;Fan, X;Mebane, C;Hamm, L;Thornton, A;Ahrendsen, JT;Anderson, MP;Christodoulou, J;Gennerich, A;Shen, Y;Chung, WK

Title:

Genotype and defects in microtubule-based motility correlate with clinical severity in KIF1Aassociated neurological disorder.

Date:

2021-04-08

Citation:

Boyle, L., Rao, L., Kaur, S., Fan, X., Mebane, C., Hamm, L., Thornton, A., Ahrendsen, J. T., Anderson, M. P., Christodoulou, J., Gennerich, A., Shen, Y. \& Chung, W. K. (2021). Genotype and defects in microtubule-based motility correlate with clinical severity in KIF1Aassociated neurological disorder.. HGG Adv, 2 (2), pp.100026-. https://doi.org/10.1016/ j.xhgg.2021.100026.

Persistent Link:

http://hdl.handle.net/11343/278068

License:

CC BY-NC-ND 